\title{
Hopf Algebra Structure of mod 2 Cohomology of Simple Lie Groups
}

\author{
By \\ Kiminao Ishitoya, ${ }^{*}$ Akira Kono* and Hirosi TODA*
}

\section{Introd uction}

The purpose of the present paper is to determine the Hopf algebra structure of the mod 2 cohomology $H^{*}\left(G ; Z_{2}\right)$ of each compact connected simple Lie group $G$. For classical type $G$, the Hopf algebra $H^{*}\left(G ; Z_{2}\right)$ is determined by Borel [6] and Baum-Browder [3], except the spinor groups $S p i n(n)$ and the semi-spinor groups $S s(4 m)$. For exceptional type $G$, it is determined by several authors [6], [8], [9], [15], except the case $G=A d E_{7}=E_{7} / Z_{2}$.

In order to describe our results, we shall use the submodule $T_{G}{ }^{*}$ of $H^{*}\left(G ; Z_{p}\right)$ which consists of the transgressive elements with respect to the fibering

$$
G \stackrel{\pi}{\rightarrow} G / T \stackrel{i}{\longrightarrow} B T
$$

where $T$ denotes a maximal torus of $G$.

The submodule $T_{G}{ }^{*}$ enjoys the following convenient properties. Let $a_{1}, \cdots, a_{l}$ be a basis of the odd dimensional part $T_{G}{ }^{\text {odd }}$ of $T_{G}{ }^{*}$, then

$$
H^{*}\left(G ; Z_{p}\right) \cong \Delta\left(a_{1}, \cdots, a_{l}\right) \otimes \operatorname{Im} \pi^{*}
$$

and

$$
T_{G}{ }^{*}=\left\langle a_{1}, \cdots, a_{l}\right\rangle+\operatorname{Im} \pi^{r},
$$

which is a part of Theorem 1.1 and the non-simply connected version of the main theorem of [16]. Furthermore, $T_{G}{ }^{*}$ is natural with respect to the group homomorphism, closed under the action of the $\bmod p$ Steenrod algebra, and each element $x$ of $T_{G}{ }^{*}$ is characterized by the diagonal

Communicated by N. Shimada, October 30, 1975.

* Department of Mathematics, Kyoto University, Kyoto. 
map:

$\bar{\phi}(x)=\phi(x)-x \otimes 1-1 \otimes x \in \operatorname{Im} \pi^{+} \otimes T_{G}{ }^{*} \quad$ (see Theorem 2.2).

The Hopf algebra structure of $H^{*}\left(G ; Z_{p}\right)$ for $G=S p i n(n), S s(4 m)$ and $A d E_{7}$ will be determined in $\S 3, \S 4$ and $\S 5$ respectively. The essential part of the results are stated as follows, for the details see Theorems $3.2,4.4$ and 5.3 :

$$
\begin{aligned}
& H^{*}\left(\operatorname{Spin}(n) ; Z_{2}\right)=\Delta\left(x_{i}, z ; 3 \leq i<n, i \neq 4,8, \cdots, 2^{s-1}\right), \\
& \left(2^{s-1}<n \leq 2^{s}, \operatorname{deg} x_{i}=i, \operatorname{deg} z=2^{s}-1, x_{i}=0 \text { if } i=2^{t} \text { or } i \geq n\right) \\
& \bar{\phi}\left(x_{i}\right)=0, \quad \bar{\phi}(z)=\sum_{i+j=2^{s-1}} x_{2 i} \otimes x_{2 j-1}, \quad S q^{1} z=\sum_{1<i<2^{s-1}} x_{2 i} x_{2 s-2 i} ; \\
& H^{*}\left(S s(4 m) ; Z_{2}\right)=\Delta\left(x_{i}, z ; 3 \leq i<4 m, i \neq 4,8, \cdots, 2^{s-1}, 2^{r}-1\right) \\
& \otimes Z_{2}[y] /\left(y^{2 r}\right), \\
& \left(4 m=2^{r} \cdot \text { odd, } 2^{s-1}<4 m \leq 2^{s}, \operatorname{deg} x_{i}=i, \operatorname{deg} z=2^{s}-1, \operatorname{deg} y=1,\right. \\
& \left.x_{i}=0 \text { if } i=2^{t} \text { or } i \geq 4 m\right) \\
& \bar{\phi}(y)=0, \quad \bar{\phi}\left(x_{i}\right)=\sum_{1<j<i / 2}\left(\begin{array}{c}
i \\
2 j
\end{array}\right) y^{2 j} \otimes x_{i-2 j}+i \cdot x_{i} \otimes y \quad\left(i \neq 2^{r}-1\right), \\
& \bar{\phi}(z)=\sum_{\substack{i+j+k=2 s-1 \\
0<i<j}}\left(\begin{array}{c}
i+j \\
j
\end{array}\right) y^{2 i} x_{2 j} \otimes x_{2 k-1}+\sum_{\substack{i+j=2 s-1-1 \\
0<i<j}} x_{2 i} x_{2 j} \otimes y, \\
& S q^{1} z=\sum_{1<i<2^{s-1}-1} x_{2 i} x_{2^{s-2 i}}+\sum_{1<i<2^{s-1}-1} y^{2} x_{2 i} x_{2 s-2 i-2} ; \\
& H^{*}\left(A d E_{7} ; Z_{2}\right)=Z_{2}\left[x_{1}, x_{5}, S q^{4} x_{5}\right] /\left(x_{1}^{4}, x_{5}^{4},\left(S q^{4} x_{5}\right)^{4}\right) \\
& \otimes \Lambda\left(S q^{1} x_{5}, x_{15}, S q^{8} S q^{4} x_{5}, S q^{8} x_{15}, S q^{4} S q^{8} x_{15}\right), \\
& \left(\operatorname{deg} x_{i}=i\right) \\
& \bar{\phi}\left(x_{1}\right)=\bar{\phi}\left(x_{5}\right)=0, \quad \bar{\phi}\left(x_{15}\right)=S q^{1} x_{5} \otimes S q^{4} x_{5}+x_{5}{ }^{2} \otimes x_{5} .
\end{aligned}
$$

\section{$\S 1$. A Transgression Theorem}

Throughout the paper, $G$ denotes a compact connected Lie group, $T$ a maximal torus of $G$, and $p$ a prime. As is seen in $\S 2$ of [16], the fibering

$$
G \stackrel{\pi}{\longrightarrow} G / T \stackrel{i}{\longrightarrow} B T
$$


is equivalent to the principal G-bundle

$$
G \stackrel{\pi}{\longrightarrow} E \stackrel{i}{\longrightarrow} B T
$$

where $E=E G \underset{T}{\times} G$ and $B T=E G \underset{T}{\times} p t$.

Denote by $T_{G}{ }^{*}$ the graded submodule of $H^{*}\left(G ; Z_{p}\right)$ which consists of the transgressive elements with respect to $(1 \cdot 1)$ or $(1 \cdot 2)$. Thus

$$
T_{G}^{*}=\delta^{-1}\left(i^{*} H^{*+1}\left(B T, p t ; Z_{p}\right)\right)
$$

for the coboundary homomorphism $\delta: H^{*}\left(G ; Z_{p}\right) \rightarrow H^{*+1}\left(E, G ; Z_{p}\right)$ and the homomorphism $i^{*}: H^{*+1}\left(B T, p t ; Z_{p}\right) \rightarrow H^{*+1}\left(E, G ; Z_{p}\right)$ induced by the projection $i$. Remark that the definition of $T_{G}{ }^{*}$ is independent of the choice of the maximal torus $T$ since any maximal tori are conjugate to each other and $G$ is connected. Obviously we have

$$
\operatorname{Im} \pi^{+} \subset T_{G}^{*} \text { for } \pi^{*}: H^{*}\left(G / T ; Z_{p}\right) \rightarrow H^{*}\left(G ; Z_{p}\right) .
$$

The following theorem has been proved for simply connected $G$ in $[16]$.

Theorem 1.1 There exist elements $a_{1}, \cdots, a_{l}$ of odd degrees such that the following assertions hold:

(i) $H^{*}\left(G ; Z_{p}\right)=\Delta\left(a_{1}, \cdots, a_{l}\right) \otimes \operatorname{Im} \pi^{*}$ as $\operatorname{Im} \pi^{*}$-modules.

(ii) $T_{G}^{*}=\left\langle a_{1}, \cdots, a_{l}\right\rangle+\operatorname{Im} \pi^{+}, \operatorname{Im} \pi^{+}=\pi^{*}\left(H^{+}\left(G / T ; Z_{p}\right)\right)$.

(iii) $H^{*}\left(G / T ; Z_{p}\right) \cong \operatorname{Im} \pi^{*} \otimes \operatorname{Im} i^{*}$ as $\operatorname{Im} i^{*}$-modules .

(iv) $\operatorname{Im} i^{*} \cong H^{*}\left(B T ; Z_{p}\right) /\left(\tau\left(a_{1}\right), \cdots, \tau\left(a_{1}\right)\right)$ for transgression images $\tau\left(a_{i}\right)$ of $a_{i}$.

(v) $\tau\left(a_{i}\right)$ are of no relation in $H^{*}\left(B T ; Z_{p}\right)$, i.e.,

$$
P\left(\operatorname{Im} i^{*}, t\right)=P\left(H^{*}\left(B T ; Z_{p}\right), t\right) \prod_{i=1}^{l}\left(1-t^{\operatorname{deg}\left(\tau\left(a_{i}\right)\right)}\right)
$$

for the Poincaré series $P\left(\sum M_{n}, t\right)=\sum \operatorname{dim} M_{n} \cdot t^{n}$.

Here $\Delta\left(a_{1}, \cdots, a_{l}\right)$ indicates the submodule spanned by the simple monomials $a_{1}^{\varepsilon_{1}} \cdots a_{l}^{\varepsilon_{l}}\left(\varepsilon_{i}=0\right.$ or 1$)$ which are linearly independent, and $\left\langle a_{i}\right\rangle$ does a submodule spanned by $a_{i}$. Remark that $l=\operatorname{rank} G$ since $H^{*}(G / T$; 
$\left.Z_{p}\right)$ is finite dimensional and $H^{*}\left(B T ; Z_{p}\right)$ is a polynomial algebra of $l$ generators of degree 2 .

The following lemma is a special case of Theorem 1.1 of [16].

Lemma 1. 2. Assume that there are elements $a_{1}, \cdots, a_{l}$ of odd degrees and a submodule $M^{*}$ of $H^{*}\left(G ; Z_{p}\right)$ satisfying the following

(i) $H^{*}\left(G ; Z_{p}\right)=\Delta\left(a_{1}, \cdots, a_{l}\right) \otimes M^{*}$ by cup products,

(ii) $M^{*} \subset \operatorname{Im} \pi^{*}$

and

(iii) $P\left(H^{*}\left(G / T ; Z_{p}\right), t\right)=P\left(M^{*}, t\right) \cdot P\left(H^{*}\left(B T ; Z_{p}\right), t\right) \cdot \prod_{i=1}^{l}\left(1-t^{\operatorname{deg} a_{i}+1}\right)$.

Then, by suitable change of generators $a_{1}, \cdots, a_{l}$ the assertions (i) -(v) of Theorem 1.1 hold.

Next we prove

Lemma 1. 3. (i) Theorem 1.1 holds for simply connected simple G.

(ii) If Theorem 1.1 holds for $G_{1}$ and $G_{2}$, then it holds for $G_{1}$ $\times G_{2}$.

(iii) Let $q$ be a prime and let $Z_{q}$ be a cyclic subgroup of order $q$ contained in the center of $G$. If Theorem 1.1 holds for $G$, then it holds for $G / Z_{q}$.

Proof. (i) follows from Propositions 3.1, 3.2 of [16].

(ii) is proved directly by use of the Künneth formula.

If $q \neq p$, then $H^{*}\left(G ; Z_{p}\right)$ is naturally isomorphic to $H^{*}\left(G / Z_{q} ; Z_{p}\right)$ and (iii) is trivial.

Let $q=p, G^{\prime}=G / Z_{q}$ and $T^{\prime}=T / Z_{q}$. Consider the cohomology spectral sequence associated with the upper fibering in the following diagram:

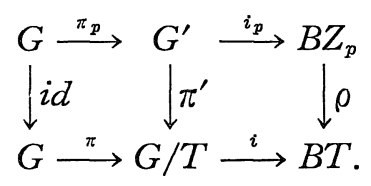


In the spectral sequence, $E_{2}{ }^{*} *=H^{*}\left(B Z_{p} ; Z_{p}\right) \otimes H^{*}\left(G ; Z_{p}\right)$ and the differential $d_{r}$ is trivial on $\operatorname{Im} \pi^{*}$ since $\operatorname{Im} \pi^{*} \subset \operatorname{Im} \pi_{p}{ }^{*}$. By the naturality of the transgression, the elements $a_{i}$ of $T_{G}{ }^{*}$ is also transgressive in this spectral sequence. Let $u$ be a generator of $H^{1}\left(B Z_{p} ; Z_{p}\right)$ then $H^{*}\left(B Z_{p}\right.$; $\left.Z_{p}\right)=\Delta(u) \otimes Z_{p}[\beta u]$.

Since $a_{i}$ is of odd degree, $\tau\left(a_{i}\right) \equiv c_{i}(\beta u)^{r}$ for some $c_{i} \in Z_{p}$ and $\operatorname{deg} a_{i}$ $=2 r-1$. If $c_{i} \equiv 0$ for all $i$, then the spectral sequence collapses, which contradicts the finiteness of $H^{*}\left(G^{\prime} ; Z_{p}\right)$. Thus $c_{k} \neq \equiv$ for some $k$, and further we may assume that $c_{i} \equiv 0$ for $i \neq k$.

By a simple computation we have

$$
E_{\infty}^{*, *}=\Delta\left(u, a_{1}, \cdots, \hat{a}_{k}, \cdots, a_{l}\right) \otimes\left(Z_{p}[\beta u] /\left((\beta u)^{r}\right) \otimes \operatorname{Im} \pi^{*}\right),
$$

for $2 r=\operatorname{deg} a_{k}+1$. Then a similar equality holds for $H^{*}\left(G^{\prime} ; Z_{p}\right)$, and the assumptions (i), (ii), (iii) of Lemma 1.2 are easily checked for $G^{\prime}$, provided that $i_{p}{ }^{*}(\beta u) \in \operatorname{Im} \pi^{* *}$. Consider the following exact and commutative diagram:

$$
\begin{aligned}
& 0 \longrightarrow H^{1}\left(B Z_{p} ; Z_{p}\right) \longrightarrow H^{2}\left(B T^{\prime} ; Z_{p}\right) \longrightarrow H^{2}\left(B T ; Z_{p}\right) \stackrel{\rho^{*}}{\longrightarrow} H^{2}\left(B Z_{p} ; Z_{p}\right) \\
& H^{2}\left(G / T ; Z_{p}\right) \stackrel{\pi^{\prime *}}{\longrightarrow} H^{2}\left(G^{\prime} ; Z_{p}\right) .
\end{aligned}
$$

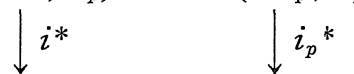

Then we see that $\rho^{*}$ is an epimorphism. Thus $i_{p}^{*}(\beta u) \in \operatorname{Im} \pi^{\prime *}$, and (iii) for the case $q=p$ follows from Lemma 1.2.

Proof of Theorem 1.1. For any compact connected Lie group $G$ there is a finite covering $\widetilde{G} \rightarrow G$ such that $\widetilde{G}$ is the product of simply connected simple Lie groups and a torus. By (i) and (ii) of Lemma 1.3, Theorem 1.1 holds for $\widetilde{G}$. The covering is divided into a sequence of coverings of prime order. Then Theorem 1.1 holds for $G$ by (iii) of Lemma 1.3.

Q.E.D.

\section{§ 2. General Arguments}

We use the following notations.

$$
T_{G}{ }^{i}=\left\{x \in T_{G}{ }^{*} ; \operatorname{deg} x=i\right\}, \quad T_{G}^{\text {even }}=\sum_{i} T_{G}{ }^{2 i}, T_{G}{ }^{\text {odd }}=\sum_{i} T_{G}{ }^{2 i+1} .
$$

Thus $T_{G}{ }^{*}=\sum_{i} T_{G}{ }^{i}=T_{G}{ }^{\text {odd }}+T_{G}{ }^{\text {even }}$ and, by (ii) of Theorem 1.1, 


$$
T_{G}^{\text {even }}=\operatorname{Im} \pi^{+} \text {and } T_{G}^{\text {odd }}=\left\langle a_{1}, \cdots, a_{l}\right\rangle \cong Z_{p}^{l} .
$$

Similarly we denote the submodule of the universally transgressive elements in $H^{+}\left(G ; Z_{p}\right)$ by

$$
U_{G}^{*}=\sum_{i} U_{G}^{i}=U_{G}^{\text {odd }}+U_{G}^{\text {even }},
$$

and that of the primitive elements by

$$
P_{G}^{*}=\sum_{i} P_{G}^{i}=P_{G}^{\text {odd }}+P_{G}^{\text {even }}=\left\{x \in H^{+}\left(G ; Z_{p}\right) ; \bar{\phi}(x)=0\right\},
$$

where

$$
\bar{\phi}(x)=\phi(x)-x \otimes 1-1 \otimes x \in H^{+}\left(G ; Z_{p}\right) \otimes H^{+}\left(G ; Z_{p}\right)
$$

for the diagonal map (comultiplication)

$$
\phi=\mu^{*}: H^{*}\left(G ; Z_{p}\right) \rightarrow H^{*}\left(G ; Z_{p}\right) \otimes H^{*}\left(G ; Z_{p}\right)
$$

induced by the group multiplication

$$
\mu: G \times G \rightarrow G
$$

identifying $H^{*}\left(G \times G ; Z_{p}\right)$ with $H^{*}\left(G ; Z_{p}\right) \otimes H^{*}\left(G ; Z_{p}\right)$ by Künneth formula.

From the naturality of the transgression and the diagonal map we have

$(2 \cdot 2)$ (i) If $f: G \rightarrow G^{\prime}$ is a homomorphism of compact connected Lie groups, $\quad f^{*} T_{G^{\prime}}{ }^{*} \subset T_{G}{ }^{*}, f^{*} U_{G^{\prime}}{ }^{*} \subset U_{G}{ }^{*}$ and $f^{*} P_{G^{\prime}}{ }^{*} \subset P_{G}{ }^{*}$.

(ii) For each cohomology operation $\alpha \in \mathcal{A}_{p}$ (the $\bmod p$ Steenrod algebra),

$$
\alpha T_{G}{ }^{*} \subset T_{G}{ }^{*}, \alpha U_{G}{ }^{*} \subset U_{G}{ }^{*} \text { and } \alpha P_{G}{ }^{*} \subset P_{G}{ }^{*}
$$

As is easily seen

$$
U_{G}{ }^{*} \subset T_{G}{ }^{*} \text { and } U_{G}{ }^{*} \subset P_{G}^{*} \text {. }
$$

From the associativity of $\mu$ it follows the (co)associativity of $\phi$ :

$$
(\phi \otimes 1) \phi=(1 \otimes \phi) \phi \text { and }(\bar{\phi} \otimes 1) \bar{\phi}=(1 \otimes \bar{\phi}) \bar{\phi} .
$$

Consider a principal $G$-bundle $G \stackrel{i}{\longrightarrow} E \stackrel{p}{\longrightarrow} B$.

Lemma 2.1. If $x \in H^{*}\left(G ; Z_{p}\right)$ is transgressive with respect to this G-bundle, $\phi(x)-x \otimes 1 \in \operatorname{Im} i^{*} \otimes H^{*}\left(G ; Z_{p}\right)$. 
Proof. Let $\bar{\mu}: E \times G \rightarrow E$ be the action of $G$ and $p_{1}: B \times G \rightarrow B$ be the projection to the first factor. Then we have the following commutative diagram:

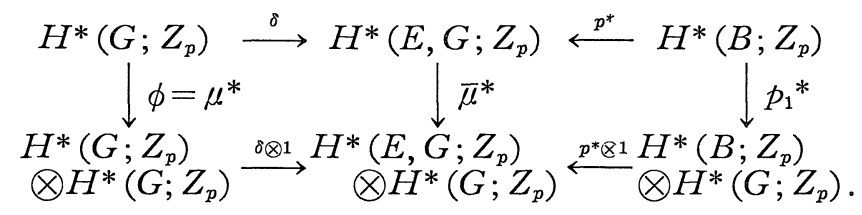

By the assumption, $\delta(x)=p^{*}(y)$ for some $y \in H^{*}\left(B ; Z_{p}\right)$, and $(\delta \otimes 1)(\phi(x)-x \otimes 1)=\bar{\mu}^{*}(\delta(x))-\delta(x) \otimes 1=\bar{\mu}^{*}\left(p^{*}(y)\right)-p^{*}(y) \otimes 1$ $=\left(p^{*} \otimes 1\right) p_{1}^{*}(y)-p^{*}(y) \otimes 1=\left(p^{*} \otimes 1\right)(y \otimes 1)-p^{*}(y) \otimes 1=0$.

Thus $\phi(x)-x \otimes 1 \in \operatorname{Ker}(\delta \otimes 1)=\operatorname{Im}\left(i^{*} \otimes 1\right)=\operatorname{Im} i^{*} \otimes H^{*}\left(G ; Z_{p}\right)$.

Q.E.D.

Remark that the lemma is valid for any associative H-space $G$ and any principal $G$-fibering.

Now we apply the above lemma to the fibering (1.1) equivalent to the principal $G$-bundle $(1 \cdot 2)$.

Theorem 2. 2. For each $x \in H^{+}\left(G ; Z_{p}\right)$, the following three conditions are equivalent:

(i) $x \in T_{G}{ }^{*}$.

(ii) $\phi(x)-x \otimes 1 \in \operatorname{Im} \pi^{*} \otimes H^{*}\left(G ; Z_{p}\right)$.

(iii) $\phi(x)-x \otimes 1 \in \operatorname{Im} \pi^{*} \otimes T_{G}^{*}$.

We shall use the following notations:

(i) $a^{I}=a_{1}^{\varepsilon_{1}} \cdots a_{l}^{\varepsilon_{l}}$ for $I=\left(\varepsilon_{1}, \cdots, \varepsilon_{l}\right)$ and $\varepsilon_{i}=0$ or $1, I+I^{\prime}$ $=\left(\varepsilon_{1}+\varepsilon_{1}{ }^{\prime}, \cdots, \varepsilon_{l}+\varepsilon_{l}{ }^{\prime}\right)$ for $I=\left(\varepsilon_{1}, \cdots, \varepsilon_{l}\right)$ and $I^{\prime}=\left(\varepsilon_{1}{ }^{\prime}, \cdots, \varepsilon_{l}{ }^{\prime}\right)$, and $|I|=\varepsilon_{1}$ $+\cdots+\varepsilon_{l}$.

(ii) $Q_{1}$ is the ideal of $H^{*}\left(G ; Z_{p}\right) \otimes H^{*}\left(G ; Z_{p}\right)$ generated by $\operatorname{Im} \pi^{+}$ $\otimes H^{*}\left(G ; Z_{p}\right)$ and $Q_{2}=H^{*}\left(G ; Z_{p}\right) \otimes \operatorname{Im} \pi^{*} \otimes H^{*}\left(G ; Z_{p}\right)$.

Lemma 2. 3. $\phi(h) \equiv 1 \otimes h \bmod Q_{1}$ for $h \in \operatorname{Im} \pi^{+}=T_{G}{ }^{\text {even }}$, 
and

$$
\phi(x) \equiv x \otimes 1+1 \otimes x \bmod Q_{1} \text { for } x \in T_{G}^{\text {odd }}
$$$$
\phi\left(a^{I}\right) \equiv \sum_{I^{\prime}+I^{\prime \prime}=I} a^{I^{\prime}} \otimes a^{I^{\prime \prime}} \bmod Q_{1} .
$$

This follows easily from Lemma 2.1 .

Proof of Theorem 2.2. Clearly (iii) implies (ii) and also (i) implies (ii) by Lemma 2.1 .

(2.5) (ii) is equivalent to $\bar{\phi}(x) \equiv 0 \bmod \operatorname{Im} \pi^{*} \otimes H^{*}\left(G ; Z_{p}\right)$, thus (ii) implies $\bar{\phi}(x) \equiv 0 \bmod Q_{1}$.

By (i) of Theorem 1.1, such $x$ is written uniquely in the form

$$
x=\sum_{I} a^{I} h_{I}+\sum_{J} \alpha_{J} a^{J} \quad\left(h_{I} \in \operatorname{Im} \pi^{+}, \alpha_{J} \in Z_{p}\right) .
$$

Then by Lemma 2.3,

$$
0 \equiv \bar{\phi}(x) \equiv \sum_{I} \sum_{\substack{I^{\prime}+I^{\prime \prime}=I \\\left|I^{\prime}\right| \neq 0}} a^{I^{\prime}} \otimes a^{I^{\prime \prime}} h_{I}+\sum_{J} \sum_{\substack{J^{\prime}+J^{\prime \prime}=J \\\left|J^{\prime}\right|,\left|J^{\prime \prime}\right| \neq 0}} \alpha_{J} a^{J^{\prime}} \otimes a^{J^{\prime \prime}} \bmod Q_{1} .
$$

This implies that $h_{I}=0$ if $|I|>0$ and $\alpha_{J}=0$ if $|J|>1$. So, $x$ satisfies (i) proving that (ii) implies (i).

Finally we prove that (ii) implies (iii) by induction on deg $x$. From the induction hypothesis we have

$$
\phi\left(h^{\prime}\right) \in \operatorname{Im} \pi^{*} \otimes \operatorname{Im} \pi^{*} \text { for } h^{\prime} \in \operatorname{Im} \pi^{+} \subset T_{G}{ }^{*} \text { with } \operatorname{deg} h^{\prime}<\operatorname{deg} x .
$$

Put $\bar{\phi}(x)=\sum_{k} h_{k}{ }^{\prime} \otimes y_{k}$ for $h_{k}{ }^{\prime} \in \operatorname{Im} \pi^{+}, \operatorname{deg} h_{k}{ }^{\prime}<\operatorname{deg} x, y_{k} \in H^{+}\left(G ; Z_{p}\right)$. By the associativity $(2 \cdot 4)$, we have

$$
\begin{aligned}
0 & =(1 \otimes \bar{\phi}) \bar{\phi}(x)-(\bar{\phi} \otimes 1) \bar{\phi}(x)=\sum_{k}\left(h_{k}{ }^{\prime} \otimes \bar{\phi}\left(y_{k}\right)-\bar{\phi}\left(h_{k}{ }^{\prime}\right) \otimes y_{k}\right) \\
& \equiv \sum_{k} h_{k}{ }^{\prime} \otimes \bar{\phi}\left(y_{k}\right) \quad \bmod Q_{2} .
\end{aligned}
$$

We may choose $\left\{h_{k}{ }^{\prime}\right\}$ linearly independent. Then $\bar{\phi}\left(y_{k}\right) \equiv 0 \bmod \operatorname{Im}$ $\pi^{*} \otimes H^{*}\left(G ; Z_{p}\right)$. So, by (2.5) $y_{k}$ satisfies (ii), and $y_{k}$ does (i). Thus

$$
\phi(x)-x \otimes 1=1 \otimes x+\sum_{k} h_{k}{ }^{\prime} \otimes y_{k} \in \operatorname{Im} \pi^{*} \otimes T_{G}{ }^{*} . \quad \text { Q.E.D. }
$$

Corollary 2. 4. $P_{G}{ }^{*} \subset T_{G}{ }^{*} . \operatorname{Im} \pi^{*}=\langle 1\rangle+T_{G}^{\text {even }}$ is a Hopf sub-algebra of $H^{*}\left(G ; Z_{p}\right)$. 
Proof. If $x \in P_{G}{ }^{*}, \phi(x)-x \otimes 1=1 \otimes x \in \operatorname{Im} \pi^{*} \otimes H^{*}\left(G ; Z_{p}\right) . \quad$ It follows $x \in T_{G}{ }^{*}$. If $x \in \operatorname{Im} \pi^{+} \subset T_{G}^{\text {even }}, \phi(x)-x \otimes 1 \in \operatorname{Im} \pi^{*} \otimes T_{G}^{\text {even }} \subset \operatorname{Im} \pi^{*}$ $\otimes \operatorname{Im} \pi^{*}$. Thus the subalgebra $\operatorname{Im} \pi^{*}$ is closed under $\phi$.

Q.E.D.

The notation

$$
\Delta_{p}\left(b_{1}, \cdots, b_{m}\right) \subset H^{*}\left(G ; Z_{p}\right)
$$

indicates the subspace having a $p$-simple system of generators $\left\{b_{1}, \cdots, b_{m}\right\}$, that is, the set $\left\{b_{1}^{\varepsilon_{l}} \cdots b_{m}{ }^{\varepsilon_{m}} ; 0 \leq \varepsilon_{i}<p\right\}$ is a $Z_{p}$-basis of $\Delta_{p}\left(b_{1}, \cdots, b_{m}\right)$. Note that

$$
\Delta_{2}\left(b_{1}, \cdots, b_{m}\right)=\Delta\left(b_{1}, \cdots, b_{m}\right) .
$$

Since $Z_{p}[b] /\left(b^{p r}\right)=\Delta_{p}\left(b, b^{p}, \cdots, b^{p r-1}\right)$, Hopf-Borel theorsm for the Hopf algebra $H^{*}\left(G ; Z_{p}\right)$ has a form:

$$
H^{*}\left(G ; Z_{p}\right)=\Delta\left(a_{1}{ }^{\prime}, \cdots, a_{l}{ }^{\prime}\right) \otimes \Delta_{p}\left(b_{1}{ }^{\prime}, \cdots, b_{m}{ }^{\prime}\right),
$$

$\operatorname{deg} a_{i}$ : odd, $\operatorname{deg} b_{j}$ : even .

Also, applying the theorem to the Hopf sub-algebra $\operatorname{Im} \pi^{*}$, we have

$$
\begin{aligned}
& H^{*}\left(G ; Z_{p}\right)=\Delta\left(a_{1}, \cdots, a_{l}\right) \otimes \Delta_{p}\left(b_{1}, \cdots, b_{m}\right), a_{i} \in T_{G}^{\text {odd }}, b_{j} \in T_{G}^{\text {even }}, \\
& \operatorname{Im} \pi^{*}=\Delta_{p}\left(b_{1}, \cdots, b_{m}\right) \text { and } T_{G}{ }^{*}=\left\langle a_{1}, \cdots, a_{l}\right\rangle+\operatorname{Im} \pi^{+} .
\end{aligned}
$$

Lemma 2.5. Given any (2.6), there exist elements $a_{i}, b_{j}$ which satisfy $(2 \cdot 6)^{\prime}$, such that $a_{i} \equiv a_{i}{ }^{\prime}, b_{j} \equiv b_{j}{ }^{\prime}(\bmod$ decomposables) and that $a_{i}=a_{i}{ }^{\prime}$ (resp. $\left.b_{j}=b_{j}{ }^{\prime}\right)$ if $a_{i}{ }^{\prime} \in T_{G}^{\text {odd }}\left(\right.$ resp. $\left.b_{j}{ }^{\prime} \in T_{G}^{\text {even }}\right)$.

This is proved by changing the generators suitably by induction on the degrees.

Lemma 2. 6. $P_{G}{ }^{\text {odd }} \subset\left\langle a_{1}, \cdots, a_{l}\right\rangle$ and $P_{G}{ }^{\text {even }} \subset\left\langle b_{1}{ }^{\prime}, \cdots, b_{m}{ }^{\prime}\right\rangle$ for some $b_{j}{ }^{\prime} \in T_{G}{ }^{\text {even }}$ such that $b_{j}{ }^{\prime} \equiv b_{j}(\bmod$ decomposables).

Proof. By Corollary 2.4, $P_{G}^{\text {odd }} \subset T_{G}^{\text {odd }}=\left\langle a_{1}, \cdots, a_{l}\right\rangle$. Consider the Hopf algebra $B=\operatorname{Im} \pi^{*}=\Delta_{p}\left(b_{1}, \cdots, b_{m}\right)$. If the elements $b_{j}$ 's are all primitive, we can show that $P(B)=P_{G}^{\text {even }}=\left\langle b_{1}, \cdots, b_{m}\right\rangle$ by the same arguments as in the proof of Theorem 2.2. Let $E^{0}(B)$ be the associated graded Hopf algebra given by the augmentation filtration $\left\{\left(B^{+}\right)^{r}\right\}$, then $E^{0}(B)$ 
is primitively generated and $P\left(E^{0}(B)\right)=\left\langle\right.$ the classes of $\left.b_{j}\right\rangle$. Since there is a natural injection of $P(B)$ into $P\left(E^{0}(B)\right)$, the second assertion follows.

Q.E.D.

\section{$\S 3$. Structure of $\boldsymbol{H}^{*}\left(\operatorname{Spin}(\boldsymbol{n}) ; \boldsymbol{Z}_{2}\right)$}

Consider the following fibering

$$
\operatorname{Spin}(n) \stackrel{\rho}{\longrightarrow} S O(n) \stackrel{\imath}{\longrightarrow} B Z_{2} \text { for } n \geq 3
$$

where $\rho$ is the universal covering and $\lambda$ is a map classifying $\rho$.

We use the following notation

$$
\begin{aligned}
& s=s(n) \text { is the integer given by } 2^{s-1}<n \leq 2^{s}, \\
& N=\left\{1,2,2^{2}, 2^{3}, \cdots\right\} .
\end{aligned}
$$

We quote the following result due to Borel [4], [5], [6].

Proposition 3. 1. (i) $H^{*}\left(S O(n) ; Z_{2}\right)=\Delta\left(\bar{x}_{1}, \bar{x}_{2}, \cdots, \bar{x}_{n-1}\right)$ for $\bar{x}_{i}$ $\in U_{\text {SO }(n)}^{i}$.

(ii) $S q^{j} \bar{x}_{i}=\left(\begin{array}{c}i \\ j\end{array}\right) \bar{x}_{i+j}(=0$ if $i+j \geq n)$, in particular $\bar{x}_{i}{ }^{2}=\bar{x}_{2 i}$.

(iii) The ideal $\operatorname{Ker} \rho^{*}$ is generated by $\bar{x}_{1}$, and $\operatorname{Im} \rho^{*}=\Delta\left(\rho^{*} \bar{x}_{i}\right.$; $i \notin N, i<n)$.

(iv) $H^{*}\left(\operatorname{Spin}(n) ; Z_{2}\right)=\operatorname{Im} \rho^{*} \otimes \Delta(z)$ for an element $z$ of $\operatorname{deg} z=2^{s}$ -1 which is transgressive with respect to the fibering (3.1) and $\tau(z) \neq 0$ in $H^{2 s}\left(B Z_{2} ; Z_{2}\right)$.

Since $\operatorname{Im} \rho^{+}$is transgressive and $\tau\left(\operatorname{Im} \rho^{+}\right)=0$ we have that

$$
\text { the element } z \text { in (iv) is determined modulo } \operatorname{Im} \rho^{*} \text {. }
$$

We put

$$
x_{i}=\rho^{*} \bar{x}_{i} \in U_{S p i n(n)}^{i} .
$$

It follows from $\rho^{*} \bar{x}_{1}=0$ and $\bar{x}_{i}{ }^{2}=\bar{x}_{2 i}$

$$
x_{i}=0 \text { if } i \in N \text { or if } i \geq n \text {. }
$$

Consider the fibering $(1 \cdot 1)$ for $G=\operatorname{Spin}(n)$ :

$$
\operatorname{Spin}(n) \stackrel{\pi}{\longrightarrow} \operatorname{Spin}(n) / T \stackrel{i}{\longrightarrow} B T \text {. }
$$

Then the structure of $H^{*}\left(\operatorname{Spin}(n) ; Z_{2}\right)$ is given by the following 
Theorem 3. 2. Put $l=[n / 2]$. There exists an element $z \in T_{\operatorname{Spin}(n)}^{2 s-1}$ such that $z \notin \operatorname{Im} \rho^{*}=\Delta\left(x_{i} ; i \notin N, i<n\right)$. Then we have the following

and

(i) $H^{*}\left(\operatorname{Spin}(n) ; Z_{2}\right)=\Delta\left(x_{3}, x_{5}, \cdots, x_{2 l-1}, z\right) \otimes \operatorname{Im} \pi^{*}$,

(ii) $T_{S p i n(n)}^{\text {odd }}=\left\langle x_{3}, x_{5}, \cdots, x_{2 l-1}, z\right\rangle$,

$$
T_{S p i n(n)}^{\text {even }}+\langle 1\rangle=\operatorname{Im} \pi^{*}=\Delta\left(x_{2 j} ; j \notin N, 2 j<n\right),
$$

(iii) $\bar{\phi}\left(x_{i}\right)=0 \quad$ and $\quad \bar{\phi}(z)=\sum_{\substack{i+j=2 s-1 \\ i>0}} x_{2 i} \otimes x_{2 j-1}$

(iv) $S q^{j} x_{i}=\left(\begin{array}{c}i \\ j\end{array}\right) x_{i+j}$, in particular $x_{i}{ }^{2}=x_{2 i}$,

$$
S q^{1}(z)=\sum_{\substack{i+j=2^{s-1} \\ i<j}} x_{2 i} x_{2 j}, \quad S q^{j} z=0 \text { for } j>1, z^{2}=0 .
$$

Note that

(3.6) the above element $z$ is unique if $n \neq 2^{s}$ and unique up to $x_{2 l-1}$ if $n=2^{s} \quad(=2 l)$.

By $(2 \cdot 3), \bar{x}_{i} \in P_{S O(n)}^{i}$ implies $x_{i} \in P_{S p i n(n)}^{i}$, i.e., $\bar{\phi}\left(x_{i}\right)=0$. By Corollary 2.4, $x_{i} \in T_{S p i n(n)}^{i}$. By Proposition 3.1,

$$
H^{*}\left(\operatorname{Spin}(n) ; Z_{2}\right)=\Delta\left(x_{2 i-1}, z ; 1<i \leq l\right) \otimes \Delta\left(x_{2 j} ; j \notin N, 2 j<n\right) .
$$

Apply Lemma 2.5, then $z$ can be changed modulo $\operatorname{Im} \rho^{*}=\Delta\left(x_{i} ; i \notin N\right.$, $i<n)$ such that $z \in T_{S p i n(n)}^{2 s} . \quad \tau(z) \neq 0$ shows that $z \notin \operatorname{Im} \rho^{*}$. Then Lemma 2.5 implies (i) and (ii) of Theorem 3.2. By the naturality of $S q^{i}$, (ii) of Proposition 3.1 implies the first assertion of (iv) of Theorem 3.2. Since $S q^{2 i} z \in T_{S p i n(n)}^{2 s+2 i-1}=0$ for $i>0, S q^{j} z=0$ for $j>1$. Thus we have obtained

(3.7) Theorem 3.2 holds except the assertion for $\bar{\phi}(z)$ and $S q^{1} z$.

Now we have

Lemma 3. 3. $P_{S p i n(n)}^{\mathrm{even}}=\left\langle x_{2 j} ; j \notin N, 2 j\langle n\rangle\right.$.

Proof. By Lemma 2.6, $\operatorname{dim} P_{S p i n(n)}^{\text {even }} \leq \operatorname{dim}\left\langle x_{2 j} ; j \notin N, 2 j\langle n\rangle\right.$. Since $x_{2 j}$ is primitive for all $j$, the equality holds.

Lemma 3. 4. $\bar{\phi}(z)=\sum_{i+j=2^{s-1}} c_{i} x_{2 i} \otimes x_{2 j-1}$ for some $c_{i} \in Z_{2}$. 

Then

Proof. By Theorem 2.2, $\bar{\phi}(z)=\sum_{j} h_{j} \otimes x_{2 j-1}$ for some $h_{j} \in \operatorname{Im} \pi^{+}$.

$$
\sum_{j} \bar{\phi}\left(h_{j}\right) \otimes x_{2 j-1}=(\bar{\phi} \otimes 1) \bar{\phi}(z)=(1 \otimes \bar{\phi}) \bar{\phi}(z)=\sum_{j} h_{j} \otimes \bar{\phi}\left(x_{2 j-1}\right)=0 .
$$

If $x_{2 j-1} \neq 0, \bar{\phi}\left(h_{j}\right)=0$. It follows from Lemma 3.3 that $h_{j}=a_{i} x_{2 i}$ for some $a_{i}$ where $2 i=2^{s}-1-(2 j-1)=2^{s}-2 j$. Of course, $a_{i}$ is arbitrary if $x_{2 j-1}=0$.

Q.E.D.

Corollary 3.5. $\bar{\phi}(z)=0$ and $S q^{1} z=0$ if $n \leq 9$ or if $n=2^{s-1}+1$.

Proof. By dimensional reason $\bar{\phi}(z)=0$ for these cases. By $(2 \cdot 2)$, (ii) and by Lemma 3.3, $S q^{1} z \in S q^{1} P_{S p i n(n)}^{2 s-1} \subset P_{S p i n(n)}^{2 s}=0$.

Lemma 3. 6. The coefficients $c_{i}$ in Lemma 3.4 satisfy $c_{i}=c_{2^{s-1}-i}$ for $i \notin N, 2 i<n / 2,2^{s}-2 i<n$, and $S q^{1} z=\sum_{i<2^{s-2}} c_{i} x_{2 i} x_{2 s-2 i}$.

Proof. Since $S q^{1} z \in T_{S p i n(n)}^{\mathrm{even}} \subset \operatorname{Im} \rho^{*}, S q^{1} z=\sum_{I} c_{I} x^{I}$ for some $c_{I} \in Z_{2}$. Since $\phi\left(x^{I}\right)=\sum_{I^{\prime}+I^{\prime \prime}=I} x^{I^{\prime}} \otimes x^{I^{\prime \prime}}$ is symmetric, so is $\bar{\phi}\left(S q^{1} z\right)$. On the other hand, $\bar{\phi}\left(S q^{1} z\right)=S q^{1} \bar{\phi}(z)=\sum_{i+j=2^{s-1}} c_{i} x_{2 i} \otimes x_{2 j}$. Thus $c_{i}=c_{2^{s-1-i}}$ if $x_{2 i} \otimes x_{2^{s-2 i}}$ $\neq 0$, and the first assertion follows. Then we have $\bar{\phi}\left(S q^{1} z\right)=\sum_{i<2^{s-2}}$ $c_{i}\left(x_{2 i} \otimes x_{2^{s-2 i}}+x_{2^{s-2 i}} \otimes x_{2 i}\right)$. So, $S q^{1} z-\sum_{i<2^{s-1}} c_{i} x_{2 i} x_{2^{s-2 i}} \in P_{S p i n(n)}^{2 s}=0$ by Lemma 3.3, and the second assertion follows.

Lemma 3. 7. Let $n=2^{s}, s \geq 4$. For some $c \in Z_{2}$, we have $\bar{\phi}(z)=c \sum_{i+j=2^{s-1}} x_{2 i} \otimes x_{2 j-1} \quad$ and $\quad S q^{1} z=c \sum_{i<2^{s-2}} x_{2 i} x_{2^{s-2 i}}$.

Proof. By (3.7), $S q^{2} z=S q^{1} x_{2 i}=0, \quad S q^{2} x_{2 i}=i \cdot x_{2 i+2} \quad$ and $S q^{2} x_{2 j-1}$ $=(j-1) x_{2 j+1}$. Thus

$$
\begin{aligned}
0 & =\bar{\phi}\left(S q^{2} z\right)=S q^{2} \bar{\phi}(z)=\sum_{i+j=2^{s-1}} c_{i}\left(i \cdot x_{2 i+2} \otimes x_{2 j-1}+(j-1) x_{2 i} \otimes x_{2 j+1}\right) \\
& =\sum_{1<k<2^{s-2}}\left(c_{2 k-1}+c_{2 k}\right)\left(x_{4 k} \otimes x_{2^{s-4 k+1}}\right) .
\end{aligned}
$$

Since $4 k \notin N$ for $2^{s-2}<2 k<2^{s-1}$, we have

$$
c_{2 k-1}=c_{2 k} \text { for } 2^{s-2}<2 k<2^{s-1} \text {. }
$$


Similarly,

$$
\begin{aligned}
0= & \bar{\phi}\left(S q^{4} z\right)=S q^{4} \bar{\phi}(z) \\
= & \sum_{i+j=2^{s-1}} c_{i}\left(\left(\begin{array}{c}
i \\
2
\end{array}\right) x_{2 i+4} \otimes x_{2 j-1}+i(j-1) x_{2 i+2} \otimes x_{2 j+1}+\left(\begin{array}{c}
j-1 \\
2
\end{array}\right) x_{2 i} \otimes x_{2 j+3}\right) \\
= & \sum_{1<k<2^{s-2}}\left\{(k-1)\left(c_{2 k-2}+c_{2 k}\right)\left(x_{4 k} \otimes x_{2^{s-4 k+3}}\right)\right. \\
& \left.+k\left(c_{2 k-3}+c_{2 k-1}\right)\left(x_{4 k-2} \otimes x_{2^{s-4 k+5}}\right)\right\},
\end{aligned}
$$

and we have

$$
c_{4 m-2}=c_{4 m} \text { and } c_{4 m-1}=c_{4 m+1} \text { for } 2^{s-2}<4 m<2^{s-1} .
$$

It follows from $(3 \cdot 8)$ and (3.9) that $c_{i-1}=c_{i}$ for $2^{s-2}+1<i<2^{s-1}$. Thus, by Lemma 3.6, $c_{i}$ is independent of $i<2^{s-1}, i \notin N$, proving Lemma 3.7 .

Next, consider the homomorphism

$$
i^{*}: H^{*}\left(\operatorname{Spin}(n) ; Z_{2}\right) \longrightarrow H^{*}\left(\operatorname{Spin}(m) ; Z_{2}\right)
$$

induced by the natural inclusion $i: \operatorname{Spin}(m) \rightarrow \operatorname{Spin}(n), m \leq n$.

Lemma 3. 8. $i^{*}\left(x_{i}\right)=x_{i}$. If $2^{s-1}<m \leq n \leq 2^{s}, i^{*}(z)=z$.

Proof. The first assertion follows from the well-known fact $\bar{i}^{*}\left(\bar{x}_{i}\right)$ $=\bar{x}_{i}$ and the commutativity of the following diagram:

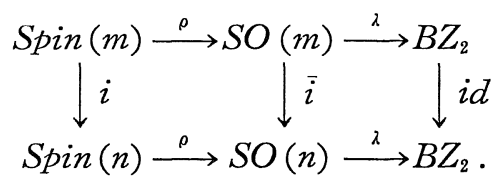

If $2^{s-1}<m \leq n \leq 2^{s}$, by (iv) of Proposition 3.1 and by the naturality of the transgression $\tau, \tau\left(i^{*}(z)\right)=\tau(z) \neq 0$. By (i) of $(2 \cdot 2)$ and by $(3 \cdot 6)$ $i^{*}(z)=z$ in $H^{2 s-1}\left(\operatorname{Spin}(m) ; Z_{2}\right)$.

Proof of Theorem 3.2. It is known that $H^{*}\left(\operatorname{Spin}\left(2^{s}\right) ; Z_{2}\right)$ is not primitively generated for $s \geq 4$ (see Kojima [7]). It follows that $c=1$ in Lemma 3.7. Apply the naturality of $\bar{\phi}$ and $S q^{1}$ to $i^{*}$ of Lemma 3.8, then we see that the formulas on $\bar{\phi}(z)$ and $S q^{1}(z)$ in Theorem 3.2 holds 
for $n>8$. Together with $(3 \cdot 7)$ and Corollary 3.5, the proof of the theorem has been established.

Remark 3. 9. In the case $n=2^{s}$, the element $z$ has not been uniquely determined. In the next $\S$, we shall see that $T_{S_{s(2 s)}^{2 s-1}}^{2 s}=\langle z\rangle$ and this is mapped injectively into $T_{S p i n\left(2^{s}\right)}^{2^{s}-1}=\left\langle z, x_{2^{s-1}}\right\rangle$ under the homomorphism $\rho_{0}^{*}$ induced by a double covering $\rho_{0}: \operatorname{Spin}\left(2^{s}\right) \longrightarrow S s\left(2^{s}\right)$. So, $z$ may be fixed as the image of $\rho_{0}^{*}$ if we fix $\rho_{0}$. However, by an automorphism of $\operatorname{Spin}\left(2^{s}\right), \rho_{0}$ is changed to another covering $\rho_{1}: \operatorname{Spin}\left(2^{s}\right) \longrightarrow S s\left(2^{s}\right)$ such that $\rho_{1}^{*}(z)=\rho_{0}^{*}(z)+x_{2^{s-1}}$.

\section{§4. Structure of $H^{*}\left(S s(n) ; Z_{2}\right), n=4 m$}

Let $n=4 m$ and $l=n / 2=2 m$. It is well known that the center of $\operatorname{Spin}(n)$ is isomorphic to $Z_{2} \times Z_{2}$. Let $a$ be the generator of the kernel of $\rho: S \operatorname{pin}(n) \longrightarrow S O(n)$ and let $b$ be another generator of the center. So, $S O(n)=\operatorname{Spin}(n) /\langle a\rangle$ and $P O(n)=\operatorname{Spin}(n) /\langle a, b\rangle$. Then the semispinor group $S s(n), n=4 m$, is defined by $S s(n)=S p i n(n) /\langle b\rangle$. By an automorphism of $\operatorname{Spin}(n), b$ is carried to $a \cdot b$. Thus

$$
S s(n)=\operatorname{Spin}(n) /\langle b\rangle \cong \operatorname{Spin}(n) /\langle a \cdot b\rangle .
$$

Note that

$$
S s(4) \cong S \operatorname{pin}(3) \times S O(3) \text { and } S s(8) \cong S O(8) .
$$

Let

$$
S s(n) \stackrel{\rho^{\prime}}{\longrightarrow} P O(n) \stackrel{x^{\prime}}{\longrightarrow} B Z_{2}
$$

be a fibering consists of a double covering $\rho^{\prime}$ and a map $\lambda^{\prime}$ classifying $\rho^{\prime}$. We use the following notations

$$
\begin{array}{ll}
s=s(n) \quad \text { for } 2^{s-1}<n \leq 2^{s}, \\
r=r(n) \quad \text { for } n=2^{r} \cdot \text { odd } \quad(r \geq 2)
\end{array}
$$

and $\bar{N}=\bar{N}(n)=N \cup\left\{2^{r}-1\right\}$ where $N=\left\{1,2,2^{2}, 2^{3}, \cdots, 2^{t}, \cdots\right\}$.

We quote the following result due to Baum-Browder [3].

Proposition 4. 1. (i) There are elements $\bar{v} \in H^{1}, x \in H^{2^{s-1}}$ and $w_{i} \in H^{i}$ for $i \neq 2^{r}-1$ such that $w_{i}=0$ for $i \in N$ or $i \geq n$ and 
$H^{*}\left(S s(n) ; Z_{2}\right)=\Delta\left(w_{i}, x ; i \notin \bar{N}, 0<i<n\right) \otimes Z_{2}[\bar{v}] /\left(\bar{v}^{2 r}\right), \bar{v}^{2 r}=0$

(ii) $S q^{j}\left(w_{i}\right)=\left\{\begin{array}{l}\bar{v}^{2 r-1} \quad \text { if } r \geq 3, j=1 \text { and } i=2^{r-1}-1, \\ \left(\begin{array}{c}i \\ j\end{array}\right) w_{i+j} \quad \text { if otherwise. }\end{array}\right.$

(iii) $\bar{\phi}\left(w_{i}\right)=\sum_{1 \leq j<i}\left(\begin{array}{l}i \\ j\end{array}\right) \bar{v}^{j} \otimes w_{i-j}$.

(iv) $w_{i}, \bar{v} \in \operatorname{Im} O^{\prime *} . \quad x$ is transgressive with respect to the fibering $(4 \cdot 2)$ and $\tau(x) \neq 0$.

In order to apply Theorem 1.1, we change the generators:

$$
\begin{aligned}
& x_{2 j-1}=w_{2 j-1}+\bar{v} w_{2 j-2} \text { for } 2 j-1 \neq 2^{r}-1,1, \\
& x_{2 j}=w_{2 j} \text { and } y=\bar{v} .
\end{aligned}
$$

Here we use the following convention

$$
x_{i}=0 \text { for } i \in \bar{N} \text { and for } i \geq n . \quad x_{0}=1 .
$$

Obviously $(l=n / 2=2 m)$,

$$
\begin{gathered}
H^{*}\left(S s(n) ; Z_{2}\right)=\Delta\left(x_{i}, x ; i \notin \bar{N}, 0<i<n\right) \otimes Z_{2}[y] /\left(y^{2 r}\right) \\
=\Delta\left(y, x_{2 j-1}, x ; 1<j \leq l, j \neq 2^{r-1}\right) \\
\otimes \Delta\left(x_{2 j} ; 2 j \notin N, 0<2 j<n\right) \otimes Z_{2}\left[y^{2}\right] /\left(y^{2 r}\right) .
\end{gathered}
$$

From the above proposition it is directly verified

Lemma 4. 2. (i) $\bar{\phi}(y)=\bar{\phi}\left(y^{2^{t}}\right)=0$,

$$
\bar{\phi}\left(x_{2 j}\right)=\sum_{1 \leq k<j}\left(\begin{array}{l}
j \\
k
\end{array}\right) y^{2 k} \otimes x_{2 j-2 k}
$$

and

$$
\bar{\phi}\left(x_{2 j-1}\right)=x_{2 j-2} \otimes y+\sum_{1 \leq k<j}\left(\begin{array}{c}
j-1 \\
k
\end{array}\right) y^{2 k} \otimes x_{2 j-2 k-1}\left(j \neq 2^{r-1}\right) .
$$

(ii) $S q^{1} x_{2 j}=0$,

$$
\begin{aligned}
& S q^{1} x_{2 j-1}=\left\{\begin{array}{l}
x_{2 j}+y^{2} x_{2 j-2} \text { for } j \neq 2^{r-2}, j \neq 2^{r-1} \text { or } r=2 \\
y^{2 r-1}+y^{2} x_{2^{r-1}-2} \text { for } j=2^{r-2}, r \geq 3
\end{array}\right. \\
& S q^{2 j} x_{i}=\left(\begin{array}{c}
i \\
2 j
\end{array}\right) x_{i+2 j}\left(i \neq 2^{r}-1\right) \text { and } S q^{j} y^{i}=\left(\begin{array}{c}
i \\
j
\end{array}\right) y^{i+j}
\end{aligned}
$$


Consider the fibering $(1 \cdot 1)$ for $G=S s(n)$ :

$$
S s(n) \stackrel{\pi}{\longrightarrow} S s(n) / T \stackrel{i}{\longrightarrow} B T .
$$

Lemma 4. 3. $y, y^{2 j}$ and $x_{i}(i \notin \bar{N})$ belong to $T_{S s(n)}^{*}$.

Proof. Each element of $H^{1}\left(S s(n) ; Z_{2}\right)$ is universally transgressive, in particular so is $y$. Thus $y \in T_{S s(n)}^{1}$. By (ii) of $(2 \cdot 2)$ and $(2 \cdot 1)$, $y^{2}=S q^{1} y \in T_{S s(n)}^{2} \subset \operatorname{Im} \pi^{*}$, and $y^{2 j} \in \operatorname{Im} \pi^{+} \subset T_{S s(n)}^{*}$. The second formula of Lemma 4.2, (i) shows that $x_{2 j}$ satisfies (ii) of Theorem 2.2. Thus $x_{2 j}$ $\in T_{S s(n)}^{*}$. Similarly it follows from the last formula of Lemma 4.2, (i) that $x_{2 j-1} \in T_{S s(n)}^{*}$.

Q.E.D.

Now applying Lemma 2.5 to (4.5), we see the existence of an element $z$ of $T_{S s(n)}^{2 s-1}$ such that $z-x \in$ decomposables $\subset \operatorname{Im} \rho^{\prime *}$. Thus $\tau(z)$ $=\tau(x) \neq 0$, and we have by Lemma 2.5 that

(4.6) the following theorem holds except the assertion for $\bar{\phi}(z)$ and $S q^{i}(z)$.

Theorem 4. 4. There exists an element $z \in T_{S s(n)}^{2 s-1}$ such that $\tau(z)$ $\neq 0$ with respect to the fibering $(4 \cdot 2)$ and that the following holds.

(i) $H^{*}\left(S s(n) ; Z_{2}\right)=\Delta\left(x_{i}, z ; i \notin \bar{N}, 0<i<n\right) \otimes Z_{2}[y] /\left(y^{2 r}\right), y^{2 r}=0$.

(ii) $T_{S s(n)}^{\text {odd }}=\left\langle y, x_{2 j-1}, z ; 1<j \leq l, j \neq 2^{r-1}\right\rangle$, $\operatorname{Im} \pi^{*}=\langle 1\rangle+T_{S s(n)}^{\mathrm{even}}=\Delta\left(x_{2 j} ; 2 j \notin N, 0<2 j<n\right) \otimes Z_{2}\left[y^{2}\right] /\left(y^{2 r}\right)$.

(iii) $\bar{\phi}$ and $S q^{i}$ for $y$ and $x_{i}$ are given in Lemma 4.2.

(iv) $\bar{\phi}(z)=\sum_{\substack{i+j+k=2 s-1 \\ j>0}}\left(\begin{array}{c}i+j \\ i\end{array}\right) y^{2 i} x_{2 j} \otimes x_{2 k-1}+\sum_{\substack{i+j=2 s-1-1 \\ 0<i<j}} x_{2 i} x_{2 j} \otimes y$, $S q^{1}(z)=\sum_{\substack{i+j=2 s-1 \\ 0<i<j}} x_{2 i} x_{2 j}+\sum_{\substack{i+j=2 s-1-1 \\ 0<i<j}} y^{2} x_{2 i} x_{2 j}$ and $\quad S q^{j}(z)=0$ for $j>1$.

The remaining part of this section is devoted to determine $\bar{\phi}(z)$ and $S q^{1}(z)$. Consider the following map between two fiberings $(3 \cdot 1)$ and 
$(4 \cdot 2)$ :

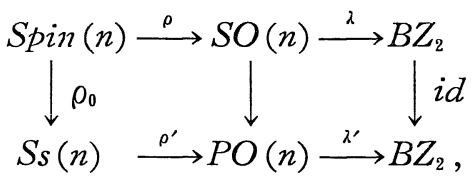

where $\rho_{0}$ is the natural projection (double covering).

Lemma 4. 5. $\rho_{0}^{*}(z)=z, \rho_{0}^{*}(y)=0$ and $\rho_{0}^{*}\left(x_{i}\right)=x_{i}$ for $i \notin \bar{N}$.

Proof. By the naturality of the transgression, $\tau\left(\rho_{0}^{*}(z)\right)=\tau(z) \neq 0$ in the upper fibering. By (i) of $(2 \cdot 2), \rho_{0}^{*}(z) \notin T_{S p i n(n)}^{2 s-1}$. Then we can take $z=\rho_{0}^{*}(z)$ in Theorem 3.2. Next consider the spectral sequence associated with the fibering

$$
\operatorname{Spin}(n) \stackrel{\rho_{0}}{\longrightarrow} S S(n) \stackrel{\lambda_{0}}{\longrightarrow} B Z_{2} .
$$

Then the only non-trivial differential is given by the transgression $\tau\left(x_{2^{r-1}}\right)$ $\neq 0$ in $H^{2 r}\left(B Z_{2} ; Z_{2}\right)$. Thus we have that the kernel of $\rho_{0}^{*}: H^{*}\left(S s(n) ; Z_{2}\right)$ $\longrightarrow H^{*}\left(\operatorname{Spin}(n) ; Z_{2}\right)$ is the ideal generated by $y$. So, $\rho_{0}^{*}(y)=0$ and $\rho_{0}^{*}\left(x_{i}\right) \neq 0$ for $i \in \bar{N}$ and $i<n$. If $i$ is odd, $\rho_{0}^{*}\left(x_{i}\right) \in T_{\operatorname{spin(n)}}^{i}=\left\langle x_{i}\right\rangle$ by (i) of $(2 \cdot 2)$ and (ii) of Theorem 3.2. Thus $\rho_{0}{ }^{*}\left(x_{i}\right)=x_{i}$ for odd $i \notin \bar{N}$. For even $i \notin \bar{N}$, by (ii) of Lemma $4.2, x_{i}=S q^{1} x_{i-1}+y \cdot f$ for some $f$. Then $\rho_{0}^{*}\left(x_{i}\right)=\rho_{0}^{*}\left(S q^{1} x_{i-1}+y \cdot f\right)=S q^{1}\left(\rho_{0}^{*}\left(x_{i-1}\right)\right)=S q^{1} x_{i-1}=x_{i} \quad$ Q.E.D.

We use a similar notation as in $\S 2$ :

(i) $x^{J}=x_{6}{ }^{\varepsilon_{3}} \cdots x_{2 j}^{\varepsilon_{j}} \cdots x_{2 l-2}^{\varepsilon_{l-1}}$ for $J=\left(\varepsilon_{3}, \cdots, \varepsilon_{j}, \cdots, \varepsilon_{l-1}\right), \varepsilon_{j}=0$ or 1 , where $x_{2 j}$ and $\varepsilon_{j}$ are omitted if $j \in N . \quad|J|=\sum \varepsilon_{j}, d(J)=\sum 2 j \varepsilon_{j} . \quad J+J^{\prime}$ $=\left(\cdots, \varepsilon_{j}+\varepsilon_{j}{ }^{\prime}, \cdots\right)$ for $J^{\prime}=\left(\cdots, \varepsilon_{j}{ }^{\prime}, \cdots\right)$.

(ii) $R_{1}$ denotes the ideal of $H^{*}\left(S s(n) ; Z_{2}\right) \otimes H^{*}\left(S s(n) ; Z_{2}\right)$ generated by $y \otimes 1 . \quad R_{2}=R_{1} \otimes H^{*}\left(S s(n) ; Z_{2}\right)$.

By Theorem 2.2, $\bar{\phi}(z) \in \operatorname{Im} \pi^{+} \otimes T_{S s(n)}^{\text {odd }}$. Then by (4.6) we have

$$
\bar{\phi}(z)=\sum_{2 i+d(J)+2 k=2^{s}} a_{i, J}^{k} y^{2 i} x^{J} \otimes x_{2 k-1}+\sum_{2 i+d(J)=2^{s-2}} b_{i, J} y^{2 i} x^{J} \otimes y
$$

for some $a_{i, J}^{k}, b_{i, J} \in Z_{2}$.

Lemma 4. 6. In (4.8) we can take $a_{0, J}^{k}=1$ for $x^{J}=x_{2^{s}-2 k}$. 
In fact, $\left(\rho_{0}^{*} \otimes \rho_{0}^{*}\right) \bar{\phi}(z)=\sum a_{0, J}^{k} x^{J} \otimes x_{2 k-1}$ coincides with $\bar{\phi}\left(\rho_{0}^{*}(z)\right)$ $=\bar{\phi}(z)=\sum_{i+j=2^{s-1}} x_{2 i} \otimes x_{2 j-1}$ in Theorem 3.2. The only question might occur to the term $x_{2^{s-2}} \otimes x_{2^{r-1}}$ which may not be in $\left(\rho_{0}^{*} \otimes \rho_{0}^{*}\right)$-image. But

$$
x_{i}=0 \text { if } i \geq 2^{s}-2^{r} \text { and } s \neq r,
$$

since $2^{s}-2^{r} \geq n=2^{r}$. odd unless $2^{s}=2^{r}=n$.

Next, we prove the following lemma by use of the associativity $(\bar{\phi} \otimes 1) \bar{\phi}=(1 \otimes \bar{\phi}) \bar{\phi}$ of $\bar{\phi}$.

Lemma 4. 7. $\bar{\phi}(z)=\sum_{\substack{i+j+k=2 s-1 \\ 0<j}}\left(\begin{array}{c}i+k-1 \\ i\end{array}\right) y^{2 i} x_{2 j} \otimes x_{2 k-1}$

$$
+\sum_{\substack{i+j=2^{s-1}-1 \\ 0<i<j}} x_{2 i} x_{2 j} \otimes y+b\left(x_{2^{s}-2} \otimes y+\sum_{\substack{i+j=2^{s-1} \\ 0<i}} y^{2 i} \otimes x_{2 j-1}\right), b \in Z_{2} .
$$

Proof. By Lemma 4.2,

$\phi\left(y^{2 i}\right) \equiv 1 \otimes y^{2 i}$ and $\phi\left(x_{2 j}\right) \equiv x_{2 j} \otimes 1+1 \otimes x_{2 j} \bmod R_{1}$. Then it follows from (4.8), modulo $R_{2}=R_{1} \otimes H^{*}\left(S s(n) ; Z_{2}\right)$,

$$
\begin{aligned}
& (\bar{\phi} \otimes 1) \bar{\phi}(z) \equiv \sum a_{i, J}^{k} \sum_{J^{\prime}+J^{\prime \prime}=J} x^{J^{\prime}} \otimes y^{2 i} x^{J^{\prime \prime}} \otimes x_{2 k-1} \\
& \quad+\sum b_{i, J} \sum_{J^{\prime}+J^{\prime \prime}=J} x^{J^{\prime}} \otimes y^{2 i} x^{J^{\prime \prime}} \otimes y \\
& \equiv(1 \otimes \bar{\phi}) \bar{\phi}(z) \equiv \sum_{k \neq 2^{r-1}} a_{0, J}^{k} x^{J} \otimes\left(x_{2 k-2} \otimes y+\sum_{1 \leq m<k}\left(\begin{array}{c}
k-1 \\
m
\end{array}\right) y^{2 m} \otimes x_{2 k-2 m-1}\right) .
\end{aligned}
$$

Comparing the coefficients, we have

$$
\begin{array}{ll}
a_{i, J}^{k}=0 & \text { if }|J|>1 \text { and } y^{2 i} x^{J} \otimes x_{2 k-1} \neq 0, \\
b_{i, J}=0 & \text { if }|J|>2 \text { and } y^{2 i} x^{J} \neq 0, \\
b_{i, J}=0 & \text { if }|J|>0, i>0 \text { and } y^{2 i} x^{J} \neq 0,
\end{array}
$$

and using Lemma 4.6,

$$
\begin{array}{r}
b_{0, J}=a_{0, I}^{j+1}=1 \text { if } x^{I}=x_{2 i}, x^{J}=x_{2 i} x_{2 j} \neq 0(i \neq j) \text { and } j+1 \neq 2^{r-1}, \\
a_{i, J}^{k}=\left(\begin{array}{c}
i+k-1 \\
i
\end{array}\right) a_{0, J}^{i+k}=\left(\begin{array}{c}
i+k-1 \\
i
\end{array}\right) \text { if } x^{J}=x_{2 j}, y^{2 i} x_{2 j} \otimes x_{2 k-1} \neq 0 \\
\text { and } i+k \neq 2^{r-1} .
\end{array}
$$

Here, $b_{0, J}=1$ for $x^{J}=x_{2 i} x_{2 j} \neq 0(i \neq j)$ since either $j+1 \neq 2^{r-1}$ or $i+1$ $\neq 2^{r-1}$. Also, if $i+k=2^{r-1}$ and $x^{J}=x_{2 j}, x_{2 j}=0$ by (4.9) and $a_{i, j}^{k} y^{2 i} x^{J}$ 
$\otimes x_{2 k-1}=0$.

Now it remains to fix the coefficients of the terms in the following

$$
x_{2^{s-2}} \otimes y, y^{2 i} \otimes x_{2^{s-2 i-1}}\left(0<i<2^{s-1}-1\right) \text { and } y^{2 s-2} \otimes y,
$$

which are all trivial or non-trivial for $n \neq 2^{s}$ or $n=2^{s}$ respectively.

Let $n=2^{s}$ and let $b, a_{i}$ and $b^{\prime}$ be the coefficients of the terms of $(4 \cdot 10)$ in (4.8) respectively. Compare the coefficients of $y^{2 i} \otimes x_{2^{s-2 i-2}}$ $\otimes y, \quad y^{2 i} \otimes y^{2 j} \otimes x_{2^{s-2 i-2 j-1}}$ and $y^{2} \otimes y^{2 s-1} \otimes y \quad$ in the equality $(\bar{\phi} \otimes 1) \bar{\phi}(z)$ $=(1 \otimes \bar{\phi}) \bar{\phi}(z)$. Then we have

$$
\begin{aligned}
& b=a_{i} \text { for } 2^{s}-2 i-2 \notin N, \\
& \left(\begin{array}{c}
i+j \\
j
\end{array}\right) a_{i+j}=\left(\begin{array}{c}
2^{s-1}-i-1 \\
j
\end{array}\right) a_{i} \text { and } b^{\prime}=0 .
\end{aligned}
$$

For even $i<2^{s-1}-2, \quad b=a_{i}=a_{i+1} . \quad$ For $i=2^{s-1}-2, \quad a_{i}=\left(\begin{array}{l}i \\ 2\end{array}\right) a_{i}=\left(\begin{array}{l}3 \\ 2\end{array}\right)$ $\cdot a_{i-2}=a_{i-2}=b$. Consequently the coefficients of the equality of the lemma are all fixed.

Q.E.D.

Lemma 4. 8. Lemma 4.7 holds for $b=0$, and

$$
S q^{1}(z)=\sum_{\substack{i+j=2 s-1 \\ 0<i<j}} x_{2 i} x_{2 j}+\sum_{\substack{i+j=2 s-1-1 \\ 0<i<j}} y^{2} x_{2 i} x_{2 j}
$$

Proof. Since $S q^{1} y=y^{2}, R_{1}$ is closed under $S q^{1}$. Consider $S q^{1}(\bar{\phi}(z))$ modulo $R_{1}$. For $j+k=2^{s-1}, k>1$, the equality $S q^{1}\left(x_{2 j} \otimes x_{2 k-1}\right)=x_{2 j} \otimes\left(x_{2 k}\right.$ $\left.+y^{2} x_{2 k-2}\right)$ holds, even for $k=2^{r-1}, 2^{r-2}$, since $x_{2 j}=0$ for $k \leq 2^{r-1}$ by (4.9). Then it follows from Lemma 4.7

$$
S q^{1}(\bar{\phi}(z)) \equiv \sum x_{2 j} \otimes\left(x_{2 k}+y^{2} x_{2 k-2}\right)+\sum_{i<j} x_{2 i} x_{2 j} \otimes y^{2}+b x_{2 s-2} \otimes y^{2} .
$$

On the other hand, $S q^{1}(z) \in S q^{1} T_{S s(n)}^{\text {odd }} \subset T_{S s(n)}^{\text {even }}=\operatorname{Im} \pi^{+}$. So, we may put

$$
S q^{1}(z)=\sum_{2 k+d(J)=2 s} c_{k, J} y^{2 k} x^{J} \text { for some } c_{k, J} \in Z_{2} .
$$

As in the proof of Lemma 4.7, we have

$$
\bar{\phi}\left(S q^{1}(z)\right) \equiv \sum c_{k, J} \sum_{J^{\prime}+J^{\prime \prime}=J} x^{J^{\prime}} \otimes y^{2 k} x^{J^{\prime \prime}} \bmod R_{1} .
$$

From the naturality $\bar{\phi}\left(S q^{1}(z)\right)=S q^{1}(\bar{\phi}(z))$, the coefficients $c_{k, J}$ of $y^{2 k} x^{J} \neq 0$ satisfy the following relations: 


$$
\begin{aligned}
& c_{k, J}=0 \text { if }|J|>2 \text { or if } k>1, \\
& c_{0, J}=c_{1, J}=1 \text { if }|J|=2 \text { and } c_{1, J}=b \text { if }|J|=1 .
\end{aligned}
$$

Thus the lemma is proved except the triviality of the coefficient $b$ of $y^{2} x_{2^{s-2}}$. If $n \neq 2^{s}, x_{2^{s-2}}=0$ and we may take $b=0$ by $(4 \cdot 10)$.

Let $n=2^{s}$, and let $Q$ be the ideal of $H^{*}\left(S s\left(2^{s}\right) ; Z_{2}\right)$ generated by the elements $x_{i}$ 's. By (ii) of Lemma $4.2, x_{i}{ }^{2}=S q^{i} x_{i}=x_{2 i}$. It follows from (ii) of Theorem 4.4 that $H^{*}\left(S s\left(2^{s}\right) ; Z_{2}\right) / Q \cong \Delta(z) \otimes Z_{2}[y] /\left(y^{2^{s}}\right)$. By (ii) of Lemma $4.2, x_{i} \equiv 0, S q^{1} x_{i} \equiv 0\left(i \neq 2^{s-1}\right)$ and $S q^{1} x_{2^{s-1-1}} \equiv y^{2^{s-1}} \bmod$ $Q$. Then we have

$$
S q^{1}(\bar{\phi}(z)) \equiv b y^{2 s-1} \otimes y^{2 s-1}, \quad \bar{\phi}\left(S q^{1}(z)\right) \equiv 0 \quad \bmod Q_{1}
$$

for the ideal $Q_{1}$ generated by $Q \otimes 1$ and $1 \otimes Q$. Thus $b=0$ completing the proof of Lemma 4.8 .

Proof of Theorem 4.4. The assertions (i), (ii) and (iii) are established by (4-6). For even $j>0, S q^{j}(z) \in T_{S s(n)}^{2 s+j-1}=0$ by (ii), and $S q^{j+1}(z)$ $=S q^{1} S q^{j}(z)=0$. Thus $S q^{j}(z)=0$ for $j>1$. The remaining part of the assertion (iv) follows from Lemma 4.8 and the following (4.11).

Q.E.D.

$$
\left(\begin{array}{c}
i+j \\
i
\end{array}\right) \equiv\left(\begin{array}{c}
i+k \\
i
\end{array}\right) \quad \bmod 2 \text { if } i+j+k=2^{t}-1
$$

For,

$$
(a+b)^{2^{t-1}} \equiv\left(a^{2 t}+b^{2 t}\right) /(a+b) \equiv \sum a^{i} b^{2 t-i-1}
$$

and $\quad(a+b+c)^{2^{t-1}} \equiv \sum(a+b)^{l} c^{2^{t-l-1}} \equiv \sum\left(\begin{array}{c}i+j \\ i\end{array}\right) a^{i} b^{j} c^{2^{t-i-j-1}}$.

Similarly

$$
(a+b+c)^{2 t-1} \equiv \sum\left(\begin{array}{c}
i+k \\
i
\end{array}\right) a^{i} b^{2 t-i-k-1} c^{k},
$$

and $(4 \cdot 11)$ follows.

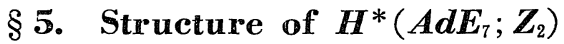

Let $E_{7}$ be the compact simply connected Lie group of type $E_{7}$. The mod 2 cohomology ring $H^{*}\left(E_{7} ; Z_{2}\right)$ is determined by Araki [1]. As is seen in [16] or by use of Theorem 1.1 we have 
Proposition 5. 1. (i) There are elements $e_{i} \in H^{i}\left(E_{7} ; Z_{2}\right)$ for $i=3,15$ such that

$$
H^{*}\left(E_{7} ; Z_{2}\right)=Z_{2}\left[e_{3}, e_{5}, e_{9}\right] /\left(e_{3}^{4}, e_{5}^{4}, e_{9}^{4}\right) \otimes \Lambda\left(e_{15}, e_{17}, e_{23}, e_{27}\right)
$$

where

$$
e_{5}=S q^{2} e_{3}, e_{9}=S q^{4} e_{5}, e_{17}=S q^{8} e_{9}, e_{23}=S q^{8} e_{15} \text { and } e_{27}=S q^{4} e_{23} .
$$

$$
T_{E_{7}}^{\text {odd }}=\left\langle e_{3}, e_{5}, e_{9}, e_{15}, e_{17}, e_{23}, e_{27}\right\rangle
$$

and

$$
\langle 1\rangle+T_{E_{7}}^{\text {even }}=\operatorname{Im} \pi^{*}=\Lambda\left(e_{3}^{2}, e_{5}^{2}, e_{9}^{2}\right) \text {. }
$$

Thomas [14] showed that $S q^{2} e_{15} \neq 0$ in $E_{6}$, and thus in $E_{7}$. So,

$$
S q^{2} e_{15}=e_{17} \text {. }
$$

The following is due to Kono-Mimura-Shimada [9] or Toda [15]:

Lemma 5. 2. $P_{E_{7}}^{15}=0$.

As is well known the center of $E_{7}$ is a cyclic group of order 2, and so denoted by $Z_{2}$. The quotient group of $E_{7}$ by the center is denoted by $A d E_{7}$, and the natural projection (double covering) by

$$
p: E_{7} \longrightarrow A d E_{7}=E_{7} / Z_{2} .
$$

We use the following notations:

$$
\begin{gathered}
e_{6}=e_{3}{ }^{2}, e_{10}=e_{5}{ }^{2} \text { and } e_{18}=e_{9}{ }^{2}, \\
M=\{1,2,5,6,9,10,15,17,18,23,27\} \\
\bar{M}=M \cup\{16,24,28\} .
\end{gathered}
$$

and

Then the results on $H^{*}\left(A d E_{7} ; Z_{2}\right)$ are summarized as follows.

Theorem 5. 3. (i) There exist elements $x_{i} \in H^{i}\left(A d E_{7} ; Z_{2}\right)$ for $i \in M$ such that $p^{*}\left(x_{i}\right)=e_{i}$ if $i \neq 1,2, x_{i}{ }^{2}=x_{2 i}$ if $2 i \in M$ and

$$
\begin{aligned}
& H^{*}\left(A d E_{7} ; Z_{2}\right)=\Delta\left(x_{i} ; i \in M\right) \\
& \quad=Z_{2}\left[x_{1}, x_{5}, x_{9}\right] /\left(x_{1}{ }^{4}, x_{5}{ }^{4}, x_{9}{ }^{4}\right) \otimes \Lambda\left(x_{6}, x_{15}, x_{17}, x_{23}, x_{27}\right) .
\end{aligned}
$$

(ii) $\quad T_{A d E_{7}}^{\text {odd }}=\left\langle x_{1}, x_{5}, x_{9}, x_{15}, x_{17}, x_{23}, x_{27}\right\rangle$

and

$$
\langle 1\rangle+T_{A d E_{7}}^{\mathrm{even}}=\operatorname{Im} \pi^{*}=\Lambda\left(x_{2}, x_{6}, x_{10}, x_{18}\right) .
$$


(iii)

$$
\begin{aligned}
& P_{A d E_{7}}^{*}=\left\langle x_{1}, x_{2}, x_{5}, x_{6}, x_{9}, x_{10}, x_{17}, x_{18}\right\rangle \\
& \bar{\phi}\left(x_{15}\right)=x_{10} \otimes x_{5}+x_{6} \otimes x_{9} \\
& \bar{\phi}\left(x_{23}\right)=x_{18} \otimes x_{5}+x_{6} \otimes x_{17}
\end{aligned}
$$

and

$$
\bar{\phi}\left(x_{27}\right)=x_{18} \otimes x_{9}+x_{10} \otimes x_{17} .
$$

(iv) For $i \in M$, the relations $S q^{j} x_{i}=\left(\begin{array}{l}i \\ j\end{array}\right) x_{i+j}$ hold, where $x_{i+j}=0$ if $i+j \notin \bar{M}, x_{18}=x_{6} x_{10}, x_{24}=x_{6} x_{18}$ and $x_{28}=x_{10} x_{18}$.

(More precise results will be seen later.)

Let $T$ be a maximal torus of $E_{7}$. As is well known the center $Z_{2}$ is a subgroup of $T$. According to Watanabe [17], we take elements $t_{1}, \cdots, t_{7}, x$ of $H^{2}(B T)$ such that

(i) $H^{*}(B T)=Z\left[t_{1}, \cdots, t_{7}, x\right] /\left(3 x-c_{1}\right), c_{1}=t_{1}+\cdots+t_{7}$,

(ii) the actions of $\Phi\left(E_{7}\right)$ on $H^{*}(B T)$ contain the permutations of $t_{i}^{\prime}$ s.

The inclusions $Z_{2} \subset T \subset E_{7}$ induce maps

$$
\iota=\iota^{\prime \prime} \circ \iota^{\prime}: B Z_{2} \stackrel{\iota^{\prime}}{\longrightarrow} B T \stackrel{\iota^{\prime \prime}}{\longrightarrow} B E_{7} \text {. }
$$

Lemma 5. 4. (i) $\iota^{\prime *}: H^{4}\left(B E_{7}\right) \longrightarrow H^{4}(B T)$ is injective and its image is generated by $c_{2}-4 x^{2}$ where $c_{2}=\sum_{1 \leq i<j \leq 7} t_{i} t_{j}$.

(ii) $\iota^{*}: H^{4}\left(B E_{7} ; Z_{2}\right) \longrightarrow H^{4}\left(B Z_{2} ; Z_{2}\right)$ is bijective.

Proof. $c^{\prime \prime}$ is a fibering with a fibre $E_{7} / T$. Since $B E_{7}$ is 3 -connected, we have a Serre exact sequence

$$
H^{3}\left(E_{7} / T\right) \longrightarrow H^{4}\left(B E_{7}\right) \stackrel{\iota^{\prime \prime}}{\longrightarrow} H^{4}(B T) \stackrel{\iota^{*}}{\longrightarrow} H^{4}\left(E_{7} / T\right) .
$$

$H^{*}\left(E_{7} / T\right)$ is given by Theorem 4.1 of [16], in particular $H^{3}\left(E_{7} / T\right)=0$ and $i^{*}$ induces an isomorphism $H^{4}(B T) /\left\langle c_{2}-4 x^{2}\right\rangle \cong H^{4}\left(E_{7} / T\right)$. Thus (i) follows. By (i) of $(5 \cdot 3), H^{*}\left(B T ; Z_{2}\right)=Z_{2}\left[t_{1}, \cdots, t_{7}\right]$. Obviously $H^{*}$ $\left(B Z_{2} ; Z_{2}\right)=Z_{2}[y], y \in H^{1}$. Put $T^{\prime}=T / Z_{2}$. From the fibering $B Z_{2} \stackrel{{ }^{\prime}}{\longrightarrow}$ $B T \longrightarrow B T^{\prime}$, we have an exact sequence $0 \longrightarrow H^{1}\left(B Z_{2} ; Z_{2}\right) \longrightarrow H^{2}\left(B T^{\prime}\right.$; $\left.Z_{2}\right) \longrightarrow H^{2}\left(B T ; Z_{2}\right) \stackrel{\iota^{\prime *}}{\longrightarrow} H^{2}\left(B Z_{2} ; Z_{2}\right)$. Since $T$ and $T^{\prime}$ are tori of the same dimension, they are isomorphic to each other. It follows easily that $\iota^{*}$ is not trivial, i.e., $\iota^{*}\left(t_{i}\right)=y^{2}$ for some $i$. Since $Z_{2}$ is the center, 
the action of the Weyl group $\Phi\left(E_{7}\right)$ is trivial on $B Z_{2}$. It follows from (ii) of $(5 \cdot 3)$ and from the naturality of the action that $\iota^{\prime *}\left(t_{1}\right)=\cdots=\iota^{\prime *}$ $\left(t_{7}\right)=y^{2}$. By (i), a generator $x_{4}$ of $H^{4}\left(B E_{7} ; Z_{2}\right) \cong Z_{2}$ is mapped onto $\iota^{\prime \prime *}\left(x_{4}\right)=c_{2}-4 x^{2}=c_{2}(\bmod 2)$. Thus

$$
\iota^{*}\left(x_{4}\right)=\iota^{\prime *}\left(c_{2}\right)=\sum_{1 \leq i<j \leq 7} \iota^{\prime *}\left(x_{i}\right) \iota^{\prime *}\left(x_{j}\right)=\left(\begin{array}{l}
7 \\
2
\end{array}\right) y^{4}=y^{4},
$$

and (ii) follows.

Q.E.D.

Consider the following fibering

$$
E_{7} \stackrel{p}{\longrightarrow} A d E_{7} \stackrel{f}{\longrightarrow} B Z_{2} .
$$

Lemma 5. 5. There exist elements $x_{i}$ of $H^{i}\left(A d E_{7} ; Z_{2}\right)$ for $i \in M$ such that

(i) $H^{*}\left(A d E_{7} ; Z_{2}\right)=\Delta\left(x_{i} ; i \in M\right)$,

(ii) $T_{A d E_{7}}^{\text {odd }}=\left\langle x_{1}, x_{5}, x_{9}, x_{15}, x_{17}, x_{23}, x_{27}\right\rangle$,

$$
\langle 1\rangle+T_{A d E_{7}}^{\mathrm{even}}=\operatorname{Im} \pi^{*}=\Delta\left(x_{2}, x_{6}, x_{10}, x_{18}\right),
$$

(iii) $x_{i}=f^{*}\left(y^{i}\right)$ for $i=1,2, p^{*}\left(x_{i}\right)=e_{i}$ for $i \neq 1,2$,

(iv) $x_{2}=S q^{1} x_{1}=x_{1}^{2}, x_{6}=S q^{1} x_{5}, x_{9}=S q^{4} x_{5}, x_{10}=S q^{5} x_{5}=x_{5}^{2}$, $x_{17}=S q^{8} x_{9}, x_{18}=S q^{9} x_{9}=x_{9}{ }^{2}, x_{23}=S q^{8} x_{15}$ and $x_{27}=S q^{4} x_{23}$.

Proof. $e_{3}$ is universally transgressive and its transgression image $x_{4}$ generates $H^{4}\left(B E_{7} ; Z_{2}\right)$. By (ii) of Lemma 5.4 , we have that the transgression $\tau$ with respect to $(5 \cdot 5)$ maps $e_{3}$ to $\tau\left(e_{3}\right)=\iota^{*}\left(x_{4}\right)=y^{4}$. The generators $e_{i}$ belong to $T_{E_{7}}^{*}$, i.e., they are transgressive with respect to the fibering

$$
E_{7} \stackrel{\pi}{\longrightarrow} E_{7} / T \longrightarrow B T \text {. }
$$

By the naturality of the transgression for the natural map of $(5 \cdot 5)$ to $(5 \cdot 5)^{\prime}, e_{i}$ 's are transgressive with respect to $(5 \cdot 5)$. Since $\tau\left(e_{3}\right)=y^{4}$, $\tau\left(e_{i}\right)=0$ for $i>3$, that is, $e_{i}=p^{*}\left(x_{i}{ }^{\prime}\right)$ for some $x_{i}{ }^{\prime}, i>3$. In the spectral sequence associated with the fibering (5.5): $E_{2}=H^{*}\left(B Z_{2} ; Z_{2}\right) \otimes H^{*}\left(E_{7}\right.$; $\left.Z_{2}\right)=Z_{2}[y] \otimes \Delta\left(e_{i}\right)$, the only non-trivial differential is $d_{4}\left(1 \otimes e_{3}\right)=y^{4} \otimes 1$. Then $E_{\infty}=Z_{2}[y] /\left(y^{4}\right) \otimes \Delta\left(e_{i} ; i \neq 3\right)$, and we have $H^{*}\left(A d E_{7} ; Z_{2}\right)=\Delta\left(x_{1}\right.$, 
$\left.x_{2}, x_{i}{ }^{\prime} ; i>3\right)$. By Lemma 2.5, we can choose $x_{i} \in T_{A d E_{7}}^{i}$ such that $x_{i} \equiv x_{i}{ }^{\prime}$ (mod decomposables) and that (i) and (ii) of the lemma hold. By (i) of $(2 \cdot 2), p^{*}\left(x_{i}\right) \in T_{E_{7}}^{i}$. By (ii) of Proposition 5.1, $T_{E_{7}}^{i}=\left\langle e_{i}\right\rangle$ for $i \in M$ and $i>3$. Thus $p^{*}\left(x_{i}\right)=e_{i}, i>3$, and (iii) of the lemma is proved.

By (ii) of this lemma, $T_{A d E_{7}}^{i}=\left\langle x_{i}\right\rangle$ for $i \in M$ and $i \neq 18$ and $T_{A d E_{7}}^{18}$ $=\left\langle x_{18}, x_{2} x_{6} x_{10}\right\rangle$. We may choose $x_{18}$ as $x_{18}=x_{9}{ }^{2}$. Since $S q^{j}$ is closed in $T_{A d E_{\eta}}^{*}$, the relation (iv) holds, up to undetermined coefficients. The coefficients are fixed by applying $p^{*}$ and comparing the coefficients in (i) of Proposition 5.1 with (i) of (5.2). For example, $p^{*}\left(x_{6}\right)=e_{6}=e_{3}{ }^{2}=S q^{3} e_{3}$ $=S q^{1} S q^{2} e_{3}=S q^{1} e_{5}=p^{*}\left(S q^{1} x_{5}\right)$, and this implies $S q^{1} x_{5}=x_{6}$. Q.E.D.

Lemma 5. 6. (i) $x_{i}^{2}=0$ for $i=2,6,10,15,17,18,23,27$.

(ii) The relation $S q^{j} x_{i}=\left(\begin{array}{l}i \\ j\end{array}\right) x_{i+j}$ in (iv) of Theorem 5.3 holds for $i=1,2,5,6,9,10,17,18$.

(iii) (iii) of Theorem 5.3 holds. $S q^{1} x_{15}=x_{6} x_{10}, S q^{1} x_{23}=x_{6} x_{18}$ and $S q^{1} x_{27}=x_{10} x_{18}$.

Proof. Obviously $x_{1} \in P_{A d E_{7}}^{1} . \quad$ By Theorem 2.2, $\bar{\phi}\left(x_{5}\right) \in \operatorname{Im} \pi^{+} \otimes T_{A d E_{7}}^{\text {odd }}$. It follows from (ii) of Lemma 5.5, $\bar{\phi}\left(x_{5}\right)=0$, i.e., $x_{5} \in P_{\boldsymbol{A} d E_{7}}^{5}$. By (ii) of (2.2), $S q^{j} P_{A d E_{\eta}}^{i} \subset P_{A d E_{7}}^{i+i}$. Then it follows from (iv) of Lemma 5.5

$$
\left\langle x_{1}, x_{2}, x_{5}, x_{6}, x_{9}, x_{10}, x_{17}, x_{18}\right\rangle \subset P_{A d E_{7}}^{*} .
$$

Also by Lemma 2.6

$$
P_{A d E_{7}}^{*} \subset\left\langle x_{i} ; i \in M\right\rangle \text {. }
$$

For $i=2,6,10,17,18, x_{i} \in P_{A d E_{7}}^{i}$ implies $x_{i}{ }^{2} \in P_{A d E_{7}}^{2 i}$ which is trivial by $(5 \cdot 6)^{\prime}: x_{i}{ }^{2}=0$. For $i=15,23,27, x_{i}{ }^{2}=S q^{i} x_{i}=S q^{1} S q^{i-1} x_{i} . \quad x_{i} \in T_{A d E_{7}}^{i}$ implies $S q^{i-1} x_{i} \in T_{A d E_{7}}^{2 i-1}$ which is trivial by (ii) of Lemma 5.5. Thus $x_{i}{ }^{2}=0$ for $i=15,23,27$, and (i) is proved.

Obviously the relation in (ii) holds for $j=0$, for $j>i$ and also for $j=i$ by (i) of this lemma and (iv) of Lemma 5.5. Let $0<j<i$ and consider the cases both that $P_{A d E_{\eta}}^{i+i}=0$ and that $\left(\begin{array}{l}i \\ j\end{array}\right) \equiv 0(\bmod 2)$ or $i+j \notin \bar{M}$. For such cases $S q^{j} x_{i}=0=\left(\begin{array}{l}i \\ j\end{array}\right) x_{i+j}$. Then, the remaining cases are the following ones:
( $\alpha) ~ j=1$ and $i=5,9,17$;
( $\beta$ ) $j=i-1$ and $i=5,9$; 


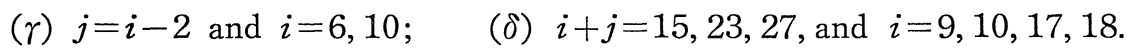

For the cases $(\alpha)$ and $(\beta)$ the relation follows from (iv) of Lemma 3.5. and the Adem relation $S q^{1} S q^{2 k}=S q^{2 k+1} \quad(i=4 k+1)$. For the case $(\gamma)$, we have $S q^{4} x_{6}=S q^{4} S q^{1} x_{5}=\left(S q^{5}+S q^{2} S q^{3}\right) x_{5}=x_{5}{ }^{2}=\left(\begin{array}{l}6 \\ 4\end{array}\right) x_{10}$ and $S q^{8} x_{10}$ $=S q^{8}\left(x_{5}{ }^{2}\right)=\left(S q^{4} x_{5}\right)^{2}=x_{9}{ }^{2}=\left(\begin{array}{c}10 \\ 8\end{array}\right) x_{18}$. For the case $(\delta),\left(\begin{array}{l}i \\ j\end{array}\right) \equiv 0(\bmod 2)$. For $i=9,17$, we have $S q^{6} x_{i}=\left(S q^{2} S q^{4}+S q^{5} S q^{1}\right) x_{i}=S q^{5} x_{i+1}$ and $S q^{5} x_{i+1}$ $=S q^{1} S q^{4} x_{i+1}=0$. We have also, $S q^{10} x_{17}=\left(S q^{2} S q^{8}+S q^{9} S q^{1}\right) x_{17}=S q^{9} x_{18}$ and $S q^{9} x_{18}=S q^{1} S q^{8} x_{18}=0$.

Consequently the relation $S q^{j} x_{i}=\left(\begin{array}{l}i \\ j\end{array}\right) x_{i+j}$ in (ii) is established.

By Theorem 2.2

$$
\bar{\phi}\left(x_{15}\right)=a x_{10} \otimes x_{5}+b x_{6} \otimes x_{9} \text { for } a, b \in Z_{2} .
$$

Since $S q^{1} x_{15} \in T_{A d E_{7}}^{16}=\left\langle x_{6} x_{10}\right\rangle, S q^{1} x_{15}=c x_{6} x_{10}$ for some $c \in Z_{2}$. Then $c\left(x_{10}\right.$ $\left.\otimes x_{6}+x_{6} \otimes x_{10}\right)=\bar{\phi} S q^{1}\left(x_{15}\right)=S q^{1} \bar{\phi}\left(x_{15}\right)=a x_{10} \otimes x_{6}+b x_{6} \otimes x_{10}$. It follows from Lemma 5.2, $a=b=c=1$. That is,

$$
\bar{\phi}\left(x_{15}\right)=x_{10} \otimes x_{5}+x_{6} \otimes x_{9} \text { and } S q^{1} x_{15}=x_{6} x_{10}=x_{16} .
$$

By use of (ii) and the Cartan formula,

$$
\bar{\phi}\left(x_{23}\right)=\bar{\phi}\left(S q^{8} x_{15}\right)=S q^{8} \bar{\phi}\left(x_{15}\right)=x_{18} \otimes x_{5}+x_{6} \otimes x_{17}
$$

and $\quad \bar{\phi}\left(x_{27}\right)=\bar{\phi}\left(S q^{4} x_{23}\right)=S q^{4} \bar{\phi}\left(x_{23}\right)=x_{18} \otimes x_{9}+x_{10} \otimes x_{17}$.

Thus $x_{15}, x_{23}, x_{27} \notin P_{A d E_{7}}^{*}$ and the equality holds in $(5 \cdot 6)$.

Finally $S q^{1} x_{23} \in T_{A d E_{7}}^{24}=\left\langle x_{6} x_{18}\right\rangle, S q^{1} x_{27} \in T_{A d E_{\eta}}^{28}=\left\langle x_{10} x_{18}\right\rangle$, and the last two formulas of (iii) are proved as above.

Q.E.D.

Lemma 5. 7. The relation $S q^{j} x_{i}=\left(\begin{array}{c}i \\ j\end{array}\right) x_{i+j}$ in (iv) of Theorem 5.3 holds for $i=15,23,27$.

Proof. First consider even $j=2 k$. Since $S q^{2 k} x_{i} \in T_{A d E_{\eta}}^{\text {odd }}$, the non-trivial cases are the following ones:

$$
S q^{2} x_{15}=x_{17}, S q^{8} x_{15}=x_{23}, S q^{4} x_{23}=x_{27} \text { and } S q^{12} x_{15}=x_{27} .
$$

The first case is reduced to $(5 \cdot 1)$ by applying $p^{*}$. The seconed and the third cases are the definitions. For the last one, $S q^{12} x_{15}=\left(S q^{4} S q^{8}\right.$ $\left.+S q^{10} S q^{2}+S q^{11} S q^{1}\right) x_{15}=S q^{4} x_{23}+S q^{10} x_{17}+S q^{11}\left(x_{6} x_{10}\right)=x_{27}$. 
Together with Lemma 5.6, we see that the formula of the lemma holds for $j=1$ and for even $j=2 k$. For odd $j=2 k+1$,

$$
S q^{j} x_{i}=S q^{1} S q^{2 k} x_{i}=\left(\begin{array}{c}
i \\
2 k
\end{array}\right) S q^{1} x_{i+2 k}=\left(\begin{array}{c}
i \\
2 k
\end{array}\right)\left(\begin{array}{c}
i+2 k \\
1
\end{array}\right) x_{i+j}=\left(\begin{array}{c}
i \\
j
\end{array}\right) x_{i+j} . \quad \text { Q.E.D. }
$$

Proof of Theorem 5.3. (i) follows from Lemma 5.5 and (i) of Lemma 5.6. Then (ii) of the theorem is (ii) of Lemma 5.5. (iii) of the theorem is (iii) of Lemma 5.6. (iv) follows from (ii) of Lemma 5.6 and Lemma 5.7.

Q.E.D.

By quite a similar but a little simpler arguments, we have

Proposition 5.8. Theorem 5.3 holds for $H^{*}\left(E_{7} ; Z_{2}\right)$ by omitting $x_{1}, x_{2}$, by adding $x_{3} \in P_{E_{1}}^{3}$ with $x_{3}{ }^{2}=x_{6}$ and by replacing $M$ by $\{3,5,6$, $9,10,15,17,18,23,27\}$.

\section{References}

[1] Araki, S., Cohomology mod 2 of the compact exceptional groups $\mathrm{E}_{6}$ and $\mathrm{E}_{7}, J$. Math. Osaka City Univ., 12 (1961), 43-65.

[2] - On cohomology mod $p$ of compact exceptional Lie groups, Ŝ́gaku, 14 (1963), 219-235 (in Japanese).

[3] Baum, P. and Browder, W., The cohomology of quotient of classical groups, Topology, 3 (1965), 305-336.

[4] Borel, A.. Sur la cohomologie des espaces fibrés principaux et des espaces homogènes de groupes de Lie compacts, Ann. of Math., 57 (1953), 115-207.

[5] - Sur l'homologie et la cohomologie des groupes de Lie compacts connexes, Amer. J. Math., 76 (1954), 273-342.

[6] - Topology of Lie groups and characteristic classes, Bull. Amer. Math. Soc., 61 (1955), 394-432.

[7] Kojima, J., On the Pontrjagin products mod 2 of spinor groups, Mem. Fac. Sci. Kyushu Univ., 11 (1957), 1-14.

[8] Kono, A. and Mimura, M., Cohomology mod 2 of the classifying spaces of the compact connected Lie group of type $\mathrm{E}_{6}, J$. Pure Appl. Algebra, 6 (1975), 61-81.

[9] Kono, A., Mimura, M. and Shimada, N., On the cohomology mod 2 of the classifying space of the 1-connected exceptional Lie groups, Aarhus Univ. Preprint Ser. 25 (1974/75).

[10] Milnor, J. and Moore, J., On the structure of Hopf algebars, Ann. of Math., 81 (1965), 211-264.

[11] Quillen, D., The mod 2 cohomology rings of extra special 2-groups and the spinor groups, Math. Ann., 194 (1971), 197-212.

[12] Serre, J.-P., Homologie singulière des espaces fibrés, Ann. of Math., 54 (1951), 425-505. 
[13] Steenrod, N. E. and Epstein, D. B. A., Cohomology operations, Ann. of Math. Studies 50, Princeton Univ. Press, 1962.

[14] Thomas, E., Exceptional Lie groups and Steenrod squares, Michigan Math. J., 11 (1964), 151-156.

[15] Toda, H., Cohomology of the classifying space of exceptional Lie groups, Manifolds Tokyo (1973), 265-271.

[16] - On the cohomology rings of some homogeneous spaces, J. Math. Kyoto Univ., 15 (1975), 185-199.

[17] Watanabe, T., The integral cohomology ring of the symmetric space EVII, J. Math. Kyoto Univ., 15 (1975), 363-385. 
